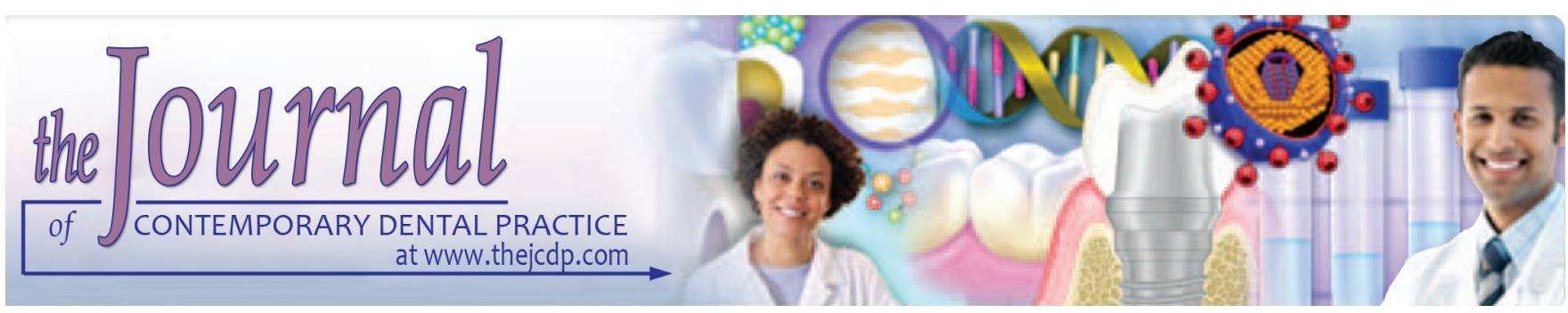

\title{
Glucose Levels and Hemodynamic Changes in Patients submitted to Routine Dental Extraction under Local Anesthesia with and without Adrenaline
}

${ }^{1}$ Sanjay Byakodi, ${ }^{2}$ Vivek Gurjar, ${ }^{3}$ Sushant Soni

\begin{abstract}
Introduction: In maxillofacial surgery, the simplest procedure that we perform is dental extraction. However, this simple procedure is challenged by the patient's poor medical condition. We generally use local anesthesia in combination with adrenaline; however, as we come across patients with diabetes mellitus and cardiovascular diseases who seek dental extraction, we need to be doubly cautious while using adrenaline. In this study, we intend to compare the effects of local anesthesia with adrenaline and local anesthesia without adrenaline on hemodynamic changes (blood pressure and pulse rate) and random blood sugar levels. The comparison is both within the group and between the two groups.
\end{abstract}

Materials and methods: Healthy patients between the ages 20 and 60 years were included and randomized into two groups of 50 each. In one group, plain local anesthesia was used, whereas in the other group, local anesthetic solution containing adrenaline was used. Medically compromised patients were excluded from the study. Random blood sugar levels, blood pressures, and pulse rates were recorded in both groups before and 10 minutes after injecting the solutions. The findings were compared.

Results: When results are compared within the group, a modest increase in the blood sugar level was noted with the group receiving local anesthetic with adrenaline. However, blood pressure and pulse rate showed no significant difference. Similarly, when between-the-groups comparison was done, not a single variable showed any significant difference.

Conclusion: The patients injected with local anesthesia containing adrenaline showed similar results to that observed in the patients injected with local anesthesia without adrenaline. However, there is a statistically significant rise in blood sugar levels when a local anesthetic is injected with adrenaline.

\footnotetext{
${ }^{1-3}$ Department of Oral and Maxillofacial Surgery, Bharati Vidyapeeth Deemed University Dental College and Hospital Sangli, Maharashtra, India

Corresponding Author: Sanjay Byakodi, Department of Oral and Maxillofacial Surgery, Bharati Vidyapeeth Deemed University Dental College and Hospital, Sangli, Maharashtra, India, Phone: +919970593949, e-mail: sanjaybyakodi@gmail.com
}

Clinical significance: Dental extractions in healthy individuals can be safely performed with local anesthetic containing adrenaline. However, in diabetic patients, it should be cautiously used.

Keywords: Adrenaline, Blood sugar level, Hemodynamics, Local anesthesia, Tooth extraction.

How to cite this article: Byakodi S, Gurjar V, Soni S. Glucose Levels and Hemodynamic Changes in Patients submitted to Routine Dental Extraction under Local Anesthesia with and without Adrenaline. J Contemp Dent Pract 2017;18(1):57-59.

Source of support: Nil

Conflict of interest: None

\section{INTRODUCTION}

Lignocaine with adrenaline is currently the most common local anesthetic agent used for tooth extraction in oral and maxillofacial surgery. The addition of adrenaline to the local anesthetic provides effective hemostasis and reduces complications caused by unnecessary bleeding. Hence, it reduces discomfort to the patient and operator. However, it has been shown that adrenaline-containing local anesthetics raise blood sugar levels in healthy individuals. Adrenaline also decreases the rate of systemic local anesthetic absorption and lowers the risk of systemic toxicity. On the contrary, local anesthetic without adrenaline provides lesser duration of action and causes more bleeding from the site and discomfort to the patient and operator. ${ }^{1}$

We intend to study and investigate values related to blood pressure, pulse rate, and blood sugar level, which are associated with vasoconstrictors in local anesthetics, and to compare the results.

\section{MATERIALS AND METHODS}

Medically fit adult patients who visited the Department of Oral and Maxillofacial Surgery, Bharati Vidyapeeth Deemed University Dental College and Hospital, Sangli, 
Maharashtra, for tooth extraction over a period of 8 months (May to December 2014) were taken for study. Proper past medical history was recorded for every patient, and patients with systemic diseases were excluded from the study. Proper consent was obtained from each patient in their known language. Patient randomization was done into two groups, viz, group I (local anesthetic with adrenaline) and group II (local anesthetic without adrenaline). Resting blood pressure, pulse rate, and random blood sugar levels were recorded for all patients before administration of both local anesthetic with adrenaline and local anesthetic without adrenaline. Then again, blood pressure, pulse rate, and random blood sugar levels were recorded 10 minutes after injection of local anesthetic. Data were analyzed using Statistical Package for the Social Sciences (SPSS), version 17, to test the significance of difference between mean values. Statistically significant value was set at $\mathrm{p}<0.05$. The study was approved by the university's ethical review committee.

\section{RESULTS}

Patients included in the study were 50 males and 50 females in number. The age range of the patients was between 20 and 60 years, with a mean of 46.81 standard deviation (SD). There were 25 males and 25 females in group I (i.e., local anesthetic with adrenaline) and 25 males and 25 females in group II (i.e., local anesthetic without adrenaline). The mean age of group I was 44.88 and that of group II was 48.74. Paired t test did not reveal any significant differences in recordings of blood pressure and pulse rate of both groups I and II (Tables 1 and 2). Statistically significant rise in blood sugar level was noted between prelocal anesthetic injection and postlocal anesthetic injection states in both the groups (Tables 1 and 2). In unpaired t test, no significant differences were found in any of the variables studied between the adrenaline and nonadrenaline groups (Table 3).

\section{DISCUSSION}

Local anesthesia is most commonly used in dental treatments. Local anesthetic with adrenaline has an important role in pain and bleeding control. It has been believed that local anesthetic with adrenaline may cause rapid rise in heart rate, blood pressure, and random blood sugar level. Adrenaline acts rapidly for a very short time. Injection of local anesthetic is associated with certain complications, and those related to the circulatory system are the majority. The presence of adrenaline in local anesthetic, with a desired property of vasoconstriction, is considered to be the major cause of these complications..$^{2-4}$ Many studies have been conducted to understand the hemodynamic changes that occur in patients undergoing treatment under local anesthesia-containing adrenaline. Some concluded that there was no change in hemodynamics,
Table 1: Group I (local anesthetic with adrenaline): Within-group comparison

\begin{tabular}{lllllll}
\hline & & $n$ & Mean & $S D$ & $t$-value & $p$-value \\
\hline Systolic & Pre & 50 & 122.32 & 14.531 & -1.204 & 0.234 \\
& Post & 50 & 124.20 & 15.071 & & \\
Diastolic & Pre & 50 & 80.28 & 8.122 & 0.000 & 1.000 \\
& Post & 50 & 80.28 & 10.999 & & \\
Pulse rate & Pre & 50 & 73.50 & 8.001 & -0.865 & 0.391 \\
& Post & 50 & 74.34 & 9.129 & & \\
Random blood & Pre & 50 & 103.32 & 24.691 & -2.873 & 0.006 \\
sugar & Post & 50 & 110.30 & 27.029 & & \\
\hline
\end{tabular}

*Paired $t$ test
Table 2: Group II (local anesthetic without adrenaline): Within-group comparison

\begin{tabular}{lllllll}
\hline & & $n$ & Mean & $S D$ & $t$-value & $p$-value \\
\hline Systolic & Pre & 50 & 124.76 & 11.262 & 1.467 & 0.149 \\
& Post & 50 & 122.52 & 16.136 & & \\
Diastolic & Pre & 50 & 81.28 & 7.470 & 1.094 & 0.279 \\
& Post & 50 & 80.52 & 7.696 & & \\
Pulse rate & Pre & 50 & 78.00 & 6.734 & -0.684 & 0.497 \\
& Post & 50 & 78.54 & 9.234 & & \\
Random & Pre & 50 & 108.52 & 23.484 & -2.360 & 0.022 \\
blood sugar & Post & 50 & 112.04 & 19.977 & & \\
\hline
\end{tabular}

*Paired $t$ test

Table 3: Between-group comparison

\begin{tabular}{|c|c|c|c|c|c|c|c|c|}
\hline & \multirow[b]{2}{*}{ Groups } & \multirow[b]{2}{*}{$n$} & \multicolumn{3}{|c|}{ Preoperative } & \multicolumn{3}{|c|}{ Postoperative } \\
\hline & & & Mean & $S D$ & $p$-value & Mean & $S D$ & $p$-value \\
\hline \multirow[t]{2}{*}{ Systolic } & I & 50 & 122.32 & 14.531 & 0.350 & 124.20 & 15.071 & 0.592 \\
\hline & II & 50 & 124.76 & 11.262 & & 122.52 & 16.136 & \\
\hline \multirow[t]{2}{*}{ Diastolic } & I & 50 & 80.28 & 8.122 & 0.523 & 80.28 & 10.999 & 0.900 \\
\hline & II & 50 & 81.28 & 7.470 & & 80.52 & 7.696 & \\
\hline \multirow[t]{2}{*}{ Pulse rate } & I & 50 & 73.50 & 8.001 & 0.003 & 74.34 & 9.129 & 0.024 \\
\hline & II & 50 & 78.00 & 6.734 & & 78.54 & 9.234 & \\
\hline \multirow[t]{2}{*}{ Random blood sugar } & I & 50 & 103.32 & 24.691 & 0.283 & 110.30 & 27.029 & 0.715 \\
\hline & II & 50 & 108.52 & 23.484 & & 112.04 & 19.977 & \\
\hline
\end{tabular}

*Unpaired $\mathrm{t}$ test 
whereas other authors reported that changes can occur depending on the dose. ${ }^{2,5,6}$ Akinmoladun et $\mathrm{al}^{2}$ suggested that patients treated with both local anesthetic with adrenaline and local anesthetic without adrenaline show similar responses in both the groups. However, in our study, increased blood sugar level has been observed when within-the-group comparison is done, while other variables show no significant difference. When Fellow et al used intravenous injections of adrenaline, they found a $30 \%$ increase in heart rate, but the values returned to preinjection levels after 15 minutes. ${ }^{7}$ A number of controversies are noted in the literature regarding variations in blood glucose levels during dental treatments. ${ }^{2,8,9}$ Tily and Thomas ${ }^{8}$ conducted a comparative study to know the pre- and postoperative changes in blood sugar levels of diabetic and healthy patients, who underwent tooth extraction under local anesthesia that contained adrenaline. They concluded that there was no noted difference in both the groups. An increase in blood glucose level was observed in both groups of our study, but there was no statistically significant difference when comparison was done between the groups.

\section{CONCLUSION}

There was no significant difference found in any of the parameters, i.e., systolic and diastolic blood pressure, pulse rate, and random blood sugar levels in both study groups. However, further studies need to be conducted to find out dose-dependent hemodynamic and blood sugar level changes in healthy individuals as well as diabetic patients.

\section{CLINICAL SIGNIFICANCE}

Dental extractions in healthy individuals can be safely performed with local anesthetic containing adrenaline. However, in diabetic patients, it should be cautiously used.

\section{ACKNOWLEDGMENT}

Authors would like to thank Dr Umesh Wadgave, Assistant Professor, Department of Public Health Dentistry, Bharati Vidyapeeth Deemed University Dental College and Hospital, Sangli, Maharashtra, for helping them in the biostatical analysis of their research project.

\section{REFERENCES}

1. Meral G, Tasar F, Sayin F, Saysel M, Kir S, Karabulut E. Effects of lidocaine with and without epinephrine on plasma epinephrine and lidocaine concentrations and hemodynamic values during third molar surgery. Oral Surg Oral Med Oral Pathol Oral Radiol Endod 2005 Aug;100(2):e25-e30.

2. Akinmoladun VI, Okoje VN, Akinosun OM, Adisa AO, Uchendu OC. Evaluation of the haemodynamic and metabolic effects of local anaesthetic agent in routine dental extractions. J Maxillofac Oral Surg 2013 Dec;12(4):424-428.

3. Someya G. Complications associated with local anesthesia. J Jpn Dent Soc 1999;27:365-373.

4. Kubota Y. Research of systemic complications during dental treatment - a review of 30 year footsteps in the Department of Dental Anesthesiology. Kokubyo Gakkai Zasshi 1994 Jun;61(2):197-206.

5. Viana AM, Campos AC, Morlin MT, Chin VK. Plasma catecholamine concentrations and hemodynamic responses to vasoconstrictor during conventional or Gow-Gates mandibular anesthesia. Oral Surg Oral Med Oral Pathol Oral Radiol Endod 2005 Oct;100(4):415-419.

6. Cáceres MT, Ludovice AC, Brito FS, Darrieux FC, Neves RS, Scanavacca MI, Sosa EA, Hachul DT. Effect of local anesthetics with and without vasoconstrictor agent in patients with ventricular arrhythmias. Arq Bras Cardiol 2008 Sep;91(3):142-147.

7. Fellows IW, Bennett T, MacDonald IA. The effect of adrenaline upon cardiovascular and metabolic functions in man. Clin Sci (Lond) 1985 Aug;69(2):215-222.

8. Tily FE, Thomas S. Glycemic effect of administration of epinephrine containing local anaesthesia in patients undergoing dental extraction: a comparison between healthy and diabetic patients. Int Dent J 2007 Apr;57(2):77-83.

9. Nakamura Y, Matsumura K, Miura K, Kurokawa H, Abe I, Takata Y. Cardiovascular and sympathetic responses to dental surgery with local anesthesia. Hypertens Res 2001 May;24(3):209-214. 